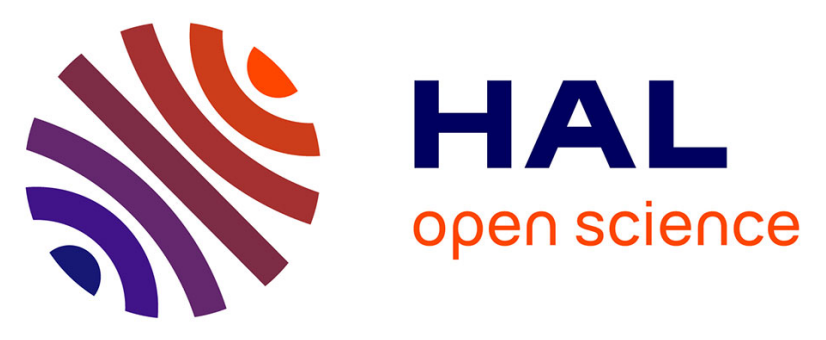

\title{
Correlated and cooperative motions in segmental relaxation: Influence of constitutive unit weight and intermolecular interactions
}

Bidur Rijal, Jorge Arturo Soto Puente, Bienvenu Atawa, Laurent Delbreilh, Kateryna Fatyeyeva, Allisson Saiter, Eric Dargent

\section{To cite this version:}

Bidur Rijal, Jorge Arturo Soto Puente, Bienvenu Atawa, Laurent Delbreilh, Kateryna Fatyeyeva, et al.. Correlated and cooperative motions in segmental relaxation: Influence of constitutive unit weight and intermolecular interactions. Physical Review E , 2016, 94 (6), pp.062502. 10.1103/PhysRevE.94.062502 . hal-02130947

\section{HAL Id: hal-02130947 https://hal.science/hal-02130947}

Submitted on 16 May 2019

HAL is a multi-disciplinary open access archive for the deposit and dissemination of scientific research documents, whether they are published or not. The documents may come from teaching and research institutions in France or abroad, or from public or private research centers.
L'archive ouverte pluridisciplinaire HAL, est destinée au dépôt et à la diffusion de documents scientifiques de niveau recherche, publiés ou non, émanant des établissements d'enseignement et de recherche français ou étrangers, des laboratoires publics ou privés. 


\title{
Correlated and cooperative motions in segmental relaxation: Influence of constitutive unit weight and intermolecular interactions
}

\author{
Bidur Rijal, ${ }^{1}$ Jorge Arturo Soto Puente, ${ }^{1,2}$ Bienvenu Atawa, ${ }^{1}$ Laurent Delbreilh, ${ }^{1}$ \\ Kateryna Fatyeyeva, ${ }^{2}$ Allisson Saiter, ${ }^{1, *}$ and Eric Dargent ${ }^{1}$ \\ ${ }^{1}$ Normandie Université, UNIROUEN, LECAP, 76000 Rouen, France \\ ${ }^{2}$ Normandie Université, UNIROUEN, INSA Rouen, CNRS, PBS, 76000 Rouen, France \\ (Received 27 September 2016; revised manuscript received 11 November 2016; published 12 December 2016)

\begin{abstract}
This work clarifies the notion of correlated and cooperative motions appearing during the $\alpha$-relaxation process through the role of the molecular weight of the constitutive units and of the interchain dipolar interactions. By studying amorphous copolymers of poly(ethylene-co-vinyl acetate) with different vinyl acetate contents, we show that the correlated motions are not sensitive to the interchain dipolar interactions, in contrast to the cooperative motions, which increase with a strengthening of the intermolecular interactions for this sample family. Concerning the influence of the molecular weight $m_{0}$, the notion of "correlated motions" seems to be equivalent to the notion of "cooperative motions" only for low $m_{0}$ systems.
\end{abstract}

DOI: 10.1103/PhysRevE.94.062502

\section{INTRODUCTION}

The dynamic nature of the glass transition is related to dynamic heterogeneities, reflected in spatial and temporal correlations of molecular motions, as well as to a distribution of the relaxation times $[1,2]$. Recent studies have extended the knowledge of the rich phenomenology of glassy systems but no unified theory is available for the moment [3-7]. Classical theories of the glass transition such as the entropic model of Adam and Gibbs (AG) [8], or the free volume approach [9], invoke the growth of the dynamic correlation size as the cause of the slowed dynamics near the glass transition temperature $T_{g}$ : Increasingly upon cooling, the constitutive units cannot reorient or diffuse independently, so that the motion of each constitutive unit depends on the neighboring motions. Thus quantifying the correlations of the molecular motions along the glass transition is essential to characterizing the motions in complex systems. This dynamic correlation size can be quantified according to the approach proposed by Berthier et al. [10] and based on the four-point dynamic susceptibility $\chi_{4}(t)$. According to Capaccioli et al. [11] the number of dynamically correlated molecules $N_{C}$ can be approximated by the following equation:

$$
N_{C}=\frac{R}{\Delta C_{p} m_{0}}\left(\frac{\beta_{\mathrm{KWW}}}{e}\right)^{2}\left(\frac{d \ln \tau_{\alpha}}{d \ln T}\right)^{2},
$$

where $m_{0}$ is the molecular weight of the constitutive unit, $\tau_{\alpha}$ the $\alpha$-relaxation time, $R$ the gas constant, $\beta_{\mathrm{KWw}}$ the stretch exponent, and $e$ the Euler's number.

Another way to quantify the correlations of the molecular motions is the approach proposed by Donth [12]. Based on calorimetric investigations and using the temperature fluctuation of the amorphous medium, it allows estimating the size of the cooperative rearranging region (CRR). Thus the number of constitutive units per CRR, noted $N_{\alpha}$ (and also called the cooperativity degree), is calculated using the

\footnotetext{
*allison.saiter@univ-rouen.fr
}

following relation [13-16]:

$$
N_{\alpha}=\frac{N_{A} k_{B} T_{\alpha}^{2} \Delta\left(1 / C_{p}\right)}{m_{0}(\delta T)^{2}},
$$

where $N_{A}$ is the Avogadro's number, $C_{p}$ the heat capacity at constant pressure, $T_{\alpha}$ the dynamic glass transition temperature, and $\delta T$ the temperature fluctuation in a CRR. Recently Saiter et al. [17] proposed an extended form of the Donth's model by combining two experimental techniques, modulated temperature differential scanning calorimetry (MTDSC) and broadband dielectric spectroscopy (BDS), enabling the calculation of the temperature dependence of the CRR size in a wide temperature range, starting from the onset of the cooperativity in the crossover region down to the $T_{g}$.

According to the recent literature [18-22], one can say that the difference between $N_{C}$ and $N_{\alpha}$, i.e., the difference between correlated motions and cooperative motions, is not clearly established. In a previous work concerning different fully amorphous polymers with different backbones, we observed a huge impact of the molecular weight of the constitutive units by comparing $N_{C}$ and $N_{\alpha}$ [7]. But the chemical structure of the studied systems was very different and we only put in evidence two tendencies related to low and high molecular weights. The goal of this work is to contribute to a better understanding of correlated and cooperative motions. For this goal, the $N_{C}$ and $N_{\alpha}$ with time and temperature variations have been studied in amorphous copolymers of poly(ethylene-co-vinyl acetate) (EVA) with different vinyl acetate content (VAc): from PVAc (100 wt \% VAc) to EVA50 (50 wt \% VAc). This sample family presents the main advantage to have the same backbone but a different number of dipolar pending groups, i.e., different interchain dipolar interactions having a clear influence on the CRR size [23].

\section{MATERIALS AND METHODS}

EVA pellets with 70,80 , and 90 wt $\%$ of VAc groups (Levapren 700, 800, and 900, respectively) were provided by Lanxess Company. Levapren 500 and 600 with 50 and 60 wt $\%$ of VAc groups, respectively, were obtained from Bayer 

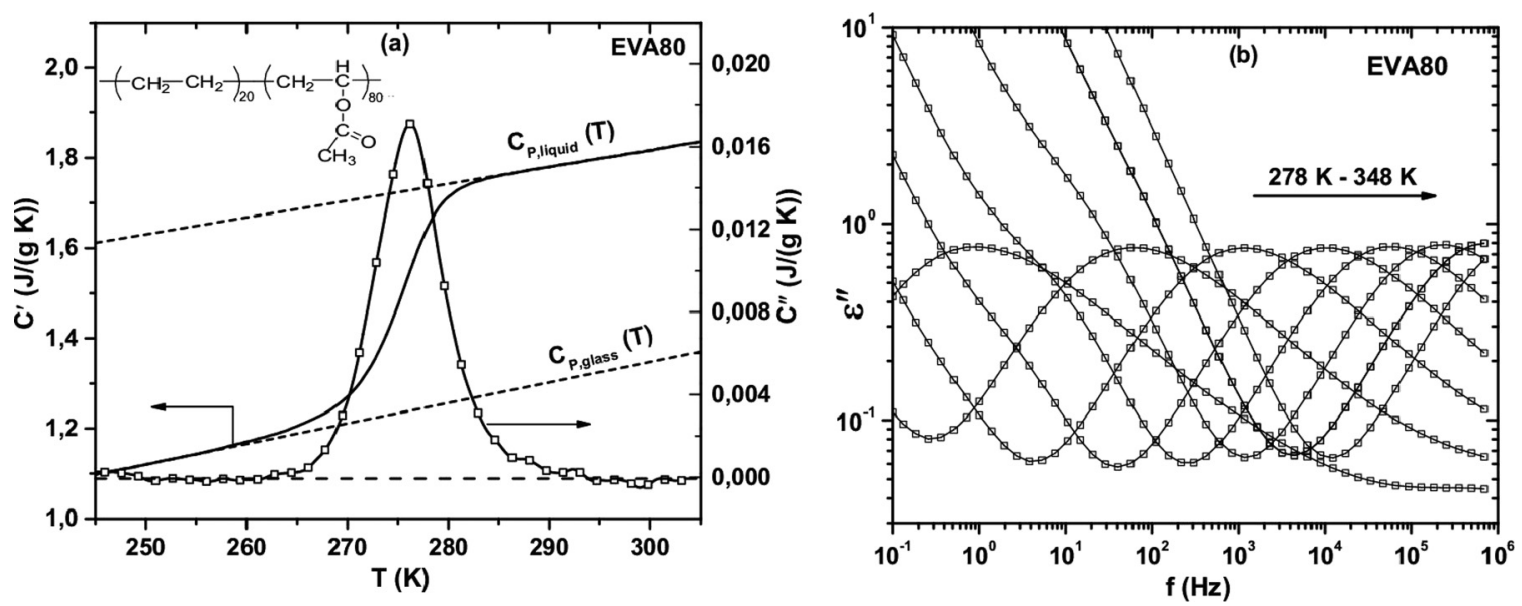

FIG. 1. EVA80 sample: (a) The real $\left(C^{\prime}\right)$ and the imaginary $\left(C^{\prime \prime}\right)$ parts of the complex heat capacity vs temperature; (b) dielectric loss $\varepsilon^{\prime \prime}$ vs frequency on the whole temperature range investigated.

Company. Samples are called "EVAxx," where $\mathrm{xx}$ is the VAc wt $\%$. PVAc pellets were provided by Sigma-Aldrich Chemical Company. The detailed procedure of the film preparation by the solution casting method from dichloromethane is given in a previous work [24]. The $m_{0}$ values of an equivalent constitutive unit for each copolymer were calculated by using the molecular weight of the VAc and the ethylene group, and the molar content $(x)$ of the VAc group.

The MTDSC experiments have been performed with a Q100 calorimeter (TA Instruments). The instrument was calibrated for heat flow, temperature, and baseline using standard $T_{\text {zero }}$ technology. The nitrogen flow was $50 \mathrm{ml} / \mathrm{min}$ for all the experiments. The calibration in temperature was carried out using standards such as indium and benzophenone, and the calibration in energy was carried out using indium. The calibration in specific heat capacity was carried out using sapphire as a reference. The sample masses were approximately $5 \mathrm{mg}$. The experiments were performed with a modulation amplitude of $\pm 1 \mathrm{~K}$, an oscillation period of $60 \mathrm{~s}$ and a heating rate of $0.5 \mathrm{~K} \mathrm{~min}^{-1}$. The BDS measurements were carried out with a Novocontrol Alpha analyzer. The samples were placed between two circular gold electrodes with a diameter of $30 \mathrm{~mm}$. The broadband dielectric converter (alpha analyzer interface) allows the measurement of the complex dielectric permittivity values (real and imaginary parts) in the frequency range from $10^{-1} \mathrm{~Hz}$ up to $10^{6} \mathrm{~Hz}$. The temperature was controlled by a Quatro Novocontrol Cryosystem with stability better than $0.2 \mathrm{~K}$.

\section{RESULTS AND DISCUSSION}

Figure 1(a) shows as an example the real $\left(C^{\prime}\right)$ and the imaginary $\left(C^{\prime \prime}\right)$ parts of the complex heat capacity for the EVA80 as a function of temperature obtained from MTDSC measurements. As expected, the $C^{\prime}$ signal shows a step at $T_{g}$, and the $C^{\prime \prime}$ signal displays a peak. Similar curves have been obtained for all the studied samples. The $T_{g}$ values range from $41.5^{\circ} \mathrm{C}$ for the PVAc, down to $-27.5^{\circ} \mathrm{C}$ for the EVA50. The details of the calorimetric investigations are given in a previous work [23].
Figure 1(b) presents as an example the dielectric loss as a function of frequency at different temperatures for the EVA80. It can be observed that the $\alpha$-relaxation peak shifts towards high frequency with the increasing temperature. At low frequencies, a strong increase of $\varepsilon^{\prime \prime}$ is observed corresponding to the dc-conductivity contribution. Similar variations have been obtained for all the studied samples.

The dielectric relaxation strength, $\Delta \varepsilon$, can be extracted from experimental relaxation data using the Havriliak-Negami fitting function [25]. Figure 2 presents the variations of the dielectric relaxation strength $\Delta \varepsilon$ as a function of the VAc content at $\tau=0.01 \mathrm{~s}$. A $\Delta \varepsilon$ decrease can be related to two main influences, a decrease of the number of dipoles and intermolecular interactions [25]. This result justifies the choice of this sample series, composed by the same polymer backbone with a progressive reduction of the number of dipoles, and thus a progressive reduction of the intermolecular interactions. Indeed, this sample series will allow discussing the evolutions

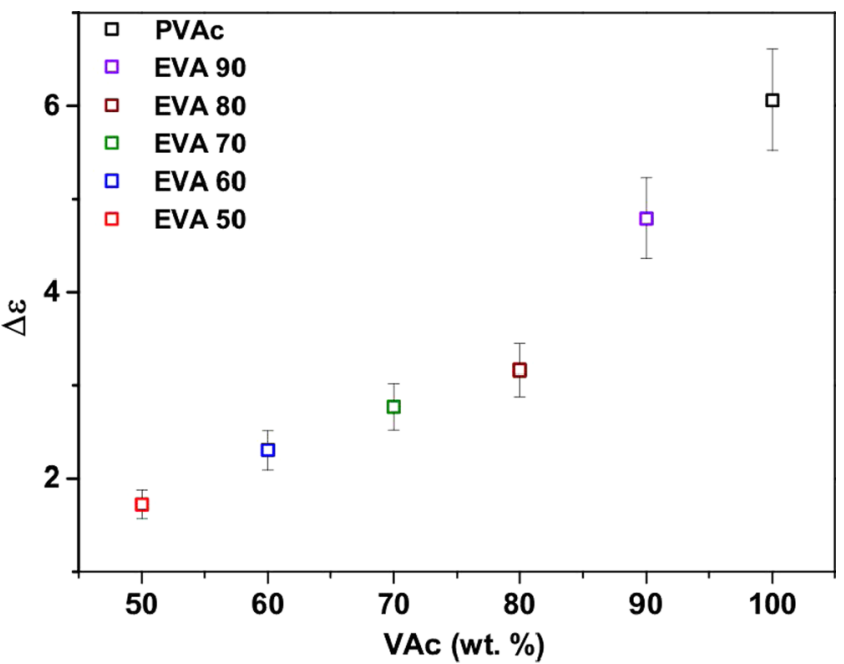

FIG. 2. Dielectric relaxation strength $(\Delta \varepsilon)$ at $\tau=0.01 \mathrm{~s}$ as a function of VAc content (wt \%). The estimated uncertainty in the amount of $\Delta \varepsilon$ is $\pm 10 \%$. 


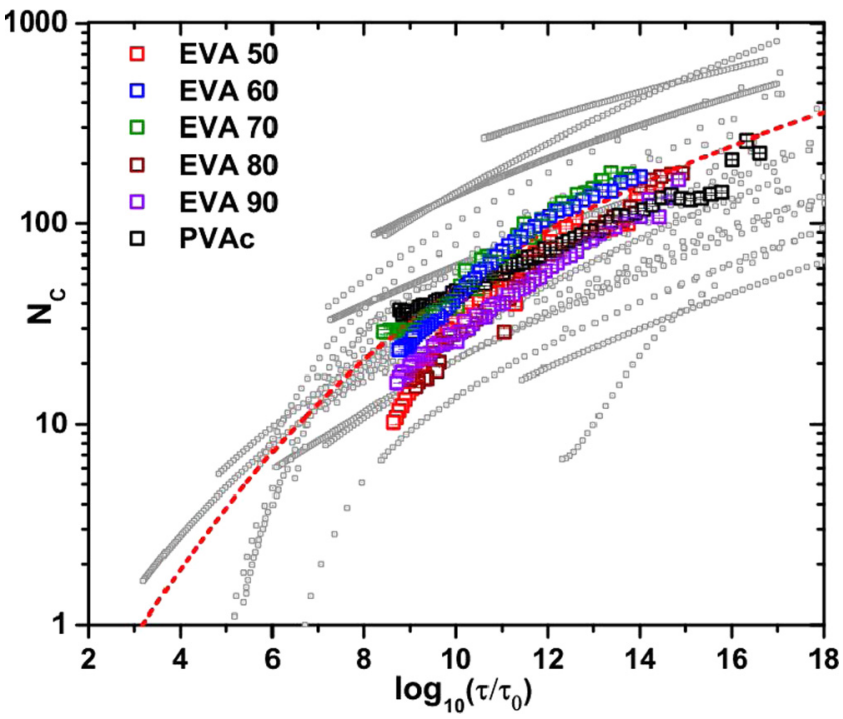

FIG. 3. Number of dynamically correlated segments $N_{C}$ for EVA copolymers as a function of $\log _{10}\left(\tau / \tau_{0}\right)$ (where $\tau_{0}=10^{-15} \mathrm{~s}$ ). The red dashed line corresponds to the fit of all the experimental points plotted using Eq. (3) with $A=4.62, N_{0}=4.0, z=1.0, \psi=0.51$. Gray squares correspond to the data in Refs. $[7,11,26]$. The estimated uncertainty in the amount of $N_{c}$ is $\pm 10 \%$.

of the correlated and the cooperative motions as a function of the degree of intermolecular interactions.

As detailed in Ref. [7], by combining MTDSC and BDS measurements, it is then possible to estimate $N_{C}$ and $N_{\alpha}$. Figure 3 shows the number of dynamically correlated segments $N_{C}$ [estimated from Eq. (1)] as a function of $\log _{10}\left(\tau / \tau_{0}\right)$ for each sample ( $\tau_{0}$ is fixed to $10^{-15} \mathrm{~s}$ ). As shown in Fig. 3 , similar $N_{C}$ variations are obtained on the whole range of the relaxation times independently from the VAc content. As classically observed in the literature for different glass formers including low molecular glass formers, polymers, and inorganic glasses $[11,26]$, the number of dynamically correlated segments $N_{C}$ increases when approaching $T_{g}$. By comparing the variations of $N_{C}$ presented in Fig. 3 to the variations obtained for more than 40 different glass formers $[7,11,26]$ (see gray squares in Fig. 3), one can note the impossibility to correlate the $N_{C}$ evolution to the number of dipolar pending groups, i.e., to the interchain dipolar interactions. Only a general tendency can be discussed for this sample family. Thus a fitting procedure allowing to follow the evolution of $N_{C}$ as a function of the relaxation time, as proposed in the literature [26,27], can be performed by considering all the data plotted in Fig. 3 . The red dashed curve in Fig. 3 corresponds to this fit using the empirical equation proposed in the literature [26,27]:

$$
\log \left(\frac{\tau}{\tau_{0}}\right)=\left(\frac{N_{C}}{N_{0}}\right)^{\psi}+z \ln \left(\frac{N_{c}}{N_{0}}\right)+\ln A .
$$

It is noticed that the fitting parameter values, i.e., $A, N_{0}, z$, and $\psi$, obtained here are quite similar to those presented in the literature for other glass formers [7,26,27]. This result confirms the idea of a common behavior for a large family of glass formers regarding the evolution of $N_{C}$ with the temperature and/or relaxation times.

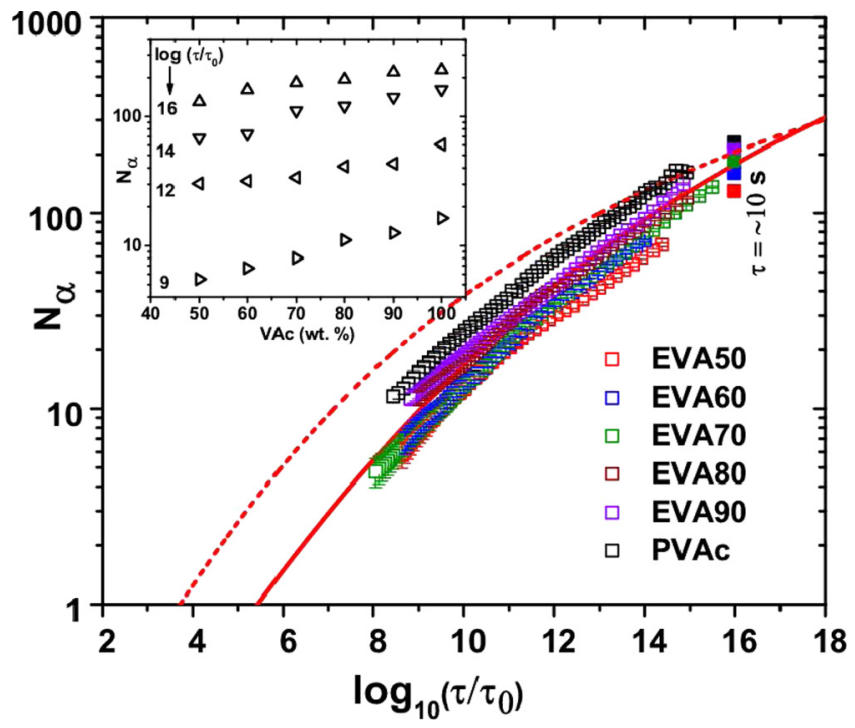

FIG. 4. $N_{\alpha}$ as a function of $\log _{10}\left(\tau / \tau_{0}\right)$ (where $\tau_{0}=10^{-15} \mathrm{~s}$ ) for EVA copolymers. Hollow symbols are from combined BDS and MTDSC experiments, and filled symbols are from only MTDSC experiments corresponding to the calorimetric $T_{g}(\tau \sim 10 \mathrm{~s})$. The red dashed line corresponds to the fit shown in Fig. 3. The solid red line corresponds to the fit of all the experimental points plotted using Eq. (3), with $A=5.62, N_{0}=3.75, z=1.1, \psi=0.45$. The inset shows the $N_{\alpha}$ variations as a function of the VAc content (wt \%) at four different relaxation times. The estimated uncertainty in the amount of $N_{\alpha}$ is $\pm 10 \%$.

Figure 4 shows the cooperativity degree $N_{\alpha}$ plotted as a function of $\tau / \tau_{0}$ with $\tau_{0}=10^{-15} \mathrm{~s}$. As expected, the cooperativity degree $N_{\alpha}$ increases with increasing of the relaxation time. Furthermore, the $N_{\alpha}$ values estimated from combined BDS and MTDSC investigations over a wide range of relaxation time are consistent with the values determined by only MTDSC investigations at the calorimetric $T_{g}(\tau \sim 10 \mathrm{~s})$. Similarly to the variations of $N_{C}$, the cooperativity degree variations are quite close for all the samples. But, as illustrated in the inset of Fig. 4 presenting the $N_{\alpha}$ variations as a function of the VAc content (wt \%) at different relaxation times, despite an uncertainty in the $N_{\alpha}$ amount of $\pm 10 \%$, the $N_{\alpha}$ values increase by increasing the VAc content (wt \%). Thus the $N_{\alpha}$ increase is related to the increase of the dipole number in the chain structure, i.e., to the strengthening of the intermolecular interactions. Earlier studies on different amorphous polymers confirm the correlation between the strength of intermolecular interactions (van der Waals) and the increase of the cooperativity degree [28,29].

In order to compare the $N_{\alpha}$ and $N_{C}$ evolutions as a function of the relaxation time, the same fitting procedure has been performed with the experimental data plotted in Fig. 4 by using Eq. (3) (see the solid red line in Fig. 4). The fit for $N_{C}$ values has been also reported (see the red dashed line in Fig. 3). Close to the calorimetric $T_{g}(\tau \sim 10 \mathrm{~s})$, similar values are obtained, but this tendency is less and less true as the relaxation time decreases. According to the two theoretical approaches used here, the temperature or the relaxation time associated with the value of $N_{C}$ or $N_{\alpha}=1$ corresponds to the limit of the cooperativity, and defines a "crossover" point in the 


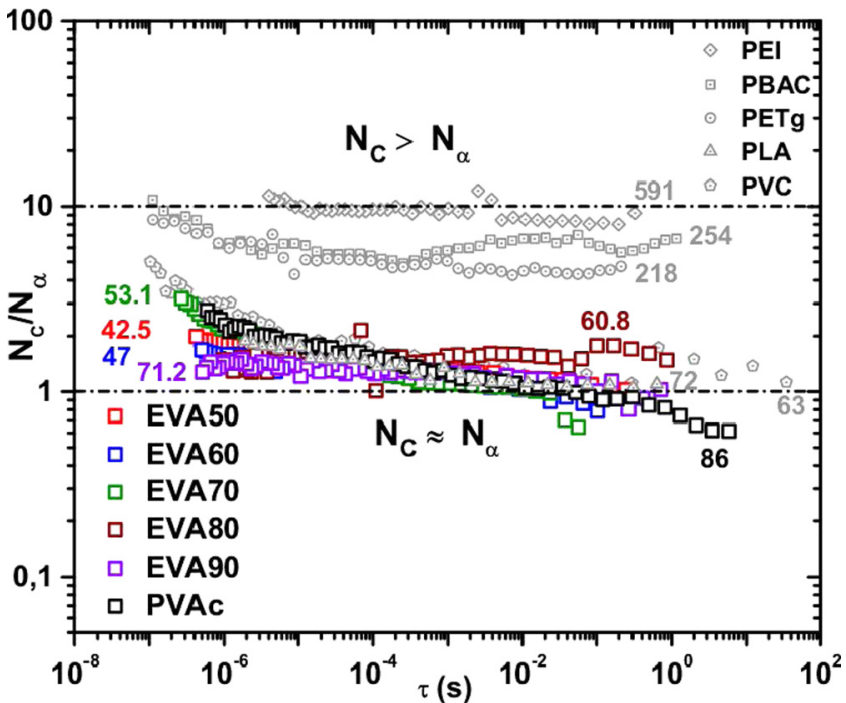

FIG. 5. $N_{C} / N_{\alpha}$ ratio as a function of the relaxation time $\tau$ for EVA copolymers. The gray symbols correspond to the data taken from Ref. [7]. The numbers indicate the molecular weight of the constitutive unit for each sample in $\mathrm{g} \mathrm{mol}^{-1}$.

Arrhenius diagram. In this work, $N_{C}=1$ at the relaxation time $\tau \sim 10^{-11} \mathrm{~s}$ and $N_{\alpha}=1$ in the range of $\tau \sim 10^{-10}-10^{-9} \mathrm{~s}$ according to the fitting curve presented in Fig. 4 for all the systems, suggesting a quite similar starting time in terms of cooperative and correlated movements when the temperature decreases.

In order to better understand the starting time in terms of cooperative and correlated movements and its relation with the molecular weight of relaxing units, the ratio $N_{C} / N_{\alpha}$ is plotted as a function of the relaxation time (Fig. 5). Indeed, as shown in a previous work [7], the starting time for cooperative and correlated movements (i.e., $N_{\alpha}$ and $N_{C}$, respectively) seems to be directly correlated to the molecular weight of the constitutive unit. Data taken from Ref. [7] for polyetherimide (PEI), poly(bisphenol A carbonate) (PBAC), poly(vinyl chloride) (PVC), copolymer poly(ethylene terephthalate glycol) (PETg), and poly(lactide acid) (PLA), are also reported in Fig. 5 in order to give an overview on the $N_{C} / N_{\alpha}$ ratio as a function of the relaxation time.

As depicted in Fig. 5, for low $m_{0}$ values, i.e., $m_{0} \leqslant$ $86 \mathrm{~g} \mathrm{~mol}^{-1}$, the $N_{C} / N_{\alpha}$ ratio is close to 1 , while for $m_{0} \geqslant$ $218 \mathrm{~g} \mathrm{~mol}^{-1}$ the $N_{C} / N_{\alpha}$ ratio is close to 7 . These original results seem to confirm the tendency evidenced in the previous work [7], i.e., that for low $m_{0}$ polymers both $N_{C}$ and $N_{\alpha}$ are quasisimilar. Furthermore, this work is consistent with the results recently obtained by Casalini et al. [20] on poly(methyl methacrylate) (PMMA). Indeed, by taking into account the uncertainty related to the different relaxation times they used to estimate $N_{C}$ and $N_{\alpha}(\tau=10 \mathrm{~s}$ and $\tau=250 \mathrm{~s}$, respectively), they found a $N_{C} / N_{\alpha}$ ratio close to 7 for bulk PMMA with $m_{0} \approx 110 \mathrm{~g} \mathrm{~mol}^{-1}$. The combination of the results presented in this work with results of the literature gives a large overview of these parameter determinations representing the amplitude of the dynamic heterogeneities in the liquidlike state. At low values of $m_{0}$ these two approaches seem to estimate dynamic heterogeneities with similar amplitude, while diverging estimations are observed for high values. The $m_{0}$ threshold seems, for the moment, quite difficult to estimate but could possibly be linked with the diverging point between monomeric unit and Kuhn length.

\section{CONCLUSION}

The structural relaxation along the glass transition has been characterized for PVAc and EVA copolymers according to two different theoretical approaches, by quantifying the number of correlated $\left(N_{C}\right)$ and cooperative $\left(N_{\alpha}\right)$ motions. This work shows for this sample family that the correlated motions are not sensitive to the interchain dipolar interactions, but a tendency appears concerning the cooperative motions: The $N_{\alpha}$ increase is related to the increase of the dipole number in the chain structure, i.e., to the strengthening of the intermolecular interactions in agreement with the literature.

Concerning the role of the molecular weight of the constitutive units, this work seems to confirm that for low $m_{0}$ systems, i.e., $m_{0} \leqslant 86 \mathrm{~g} \mathrm{~mol}^{-1}$, the notion of "correlated motions" seems to be equivalent to the notion of "cooperative motions" not only in terms of time scale at which these motions appear, but also in terms of number of constitutive units implied during the $\alpha$ process. However, this equivalence seems not to be valid for the systems with high $m_{0}$ values $\left(m_{0} \geqslant 218 \mathrm{~g} \mathrm{~mol}^{-1}\right)$ as is well illustrated in the graphical abstract.

\section{ACKNOWLEDGMENTS}

The authors are grateful to the GRR Matériaux (Normandy Region, France) for the financial support for the Ph.D. thesis and BDS equipment, and to the Lanxess Company for the donation of the EVA pellets. The authors declare no competing financial interest.
[1] L. Berthier and G. Biroli, Theoretical perspective on the glass transition and amorphous materials, Rev. Mod. Phys. 83, 587 (2011).

[2] D. Fragiadakis, R. Casalini, and C. M. Roland, Comparing dynamic correlation lengths from an approximation to the four-point dynamic susceptibility and from the picosecond vibrational dynamics, Phys. Rev. E 84, 042501 (2011).
[3] K. L. Ngai and C. M. Roland, Note: Thermorheological complexity in polymers and the problem of the glass transition, J. Chem. Phys. 139, 036101 (2013).

[4] G. Biroli and J. P. Garrahan, Perspective: The glass transition, J. Chem. Phys. 138, 12A301 (2013).

[5] F. H. Stillinger and P. G. Debenedetti, Glass transition thermodynamics and kinetics, Annu. Rev. Condens. Matter Phys. 4, 263 (2013). 
[6] D. Fragiadakis and C. M. Roland, Dynamic correlations and heterogeneity in the primary and secondary relaxations of a model molecular liquid, Phys. Rev. E 89, 052304 (2014).

[7] B. Rijal, L. Delbreilh, and A. Saiter, Dynamic heterogeneity and cooperative length scale at dynamic glass transition in glass forming liquids, Macromolecules 48, 8219 (2015).

[8] G. Adam and J. H. Gibbs, On the temperature dependence of cooperative relaxation properties in glass-forming liquids, J. Chem. Phys. 43, 139 (1965).

[9] D. Turnbull and M. H. Cohen, Free-volume model of the amorphous phase: glass transition, J. Chem. Phys. 34, 120 (1961).

[10] L. Berthier, G. Biroli, J.-P. Bouchaud, L. Cipelletti, and W. van Saarloos, Dynamical Heterogeneities in Glasses, Colloids, and Granular Media (Oxford University Press, Oxford, (2011).

[11] S. Capaccioli, G. Ruocco, and F. Zamponi, Dynamically correlated regions and configurational entropy in supercooled liquids, J. Phys. Chem. B 112, 10652 (2008).

[12] E.-J. Donth, The Glass Transition: Relaxation Dynamics in Liquids and Disordered Materials (Springer Science \& Business Media, New York, 2001).

[13] A. Saiter, J.-M. Saiter, R. Golovchak, M. Shpotyuk, and O. Shpotyuk, Cooperative rearranging region size and free volume in As-Se glasses, J. Phys.: Condens. Matter 21, 075105 (2009).

[14] N. Delpouve, C. Lixon, A. Saiter, E. Dargent, and J. Grenet, Amorphous phase dynamics at the glass transition in drawn semi-crystalline polyester: DMA and TMDSC comparative studies, J. Therm. Anal. Calorim. 97, 541 (2009).

[15] F. Hamonic, D. Prevosto, E. Dargent, and A. Saiter, Contribution of chain alignment and crystallization in the evolution of cooperativity in drawn polymers, Polymer 55, 2882 (2014).

[16] E. Bouthegourd, A. Esposito, D. Lourdin, A. Saiter, and J.-M. Saiter, Size of the cooperative rearranging regions vs. fragility in complex glassy systems: Influence of the structure and the molecular interactions, Physica B 425, 83 (2013).

[17] A. Saiter, L. Delbreilh, H. Couderc, K. Arabeche, A. Schönhals, and J.-M Saiter, Temperature dependence of the characteristic length scale for glassy dynamics: Combination of dielectric and specific heat spectroscopy, Phys. Rev. E 81, 041805 (2010).
[18] D. Fragiadakis, R. Casalini, R. B. Bogoslovov, C. G. Robertson, and C. M. Roland, Dynamic heterogeneity and density scaling in 1,4-polyisoprene, Macromolecules 44, 1149 (2011).

[19] R. Casalini, D. Fragiadakis, and C. M. Roland, Relaxation dynamics of poly(methyl acrylate) atelevated pressure, Macromolecules 44, 6928 (2011).

[20] R. Casalini, L. Zhu, E. Baer, and C. M. Roland, Segmental dynamics and the correlation length in nanoconfined PMMA, Polymer 88, 133 (2016).

[21] M. Pieruccini, A. Alessandrini, S. Sturniolo, M. Corti, and A. Rigamenti, Small and large scale segmental motion in polymers: estimating cooperativity length by ordinary relaxation experiments, Polym. Int. 64, 1506 (2015).

[22] M. Pieruccini and A. Alessandrini, Method for estimating the cooperativity length in polymers, Phys. Rev. E 91, 052603 (2015).

[23] J. A. S. Puente, B. Rijal, L. Delbreilh, K. Fatyeyeva, A. Saiter, and E. Dargent, Segmental mobility and glass transition of poly(ethylene-vinyl acetate) copolymers: Is there a continuum in the dynamic glass transitions from PVAc to PE? Polymer 76, 213 (2015).

[24] J. A. S. Puente, K. Fatyeyeva, S. Marais, and E. Dargent, Multifunctional hydrolyzed EVA membranes with tunable microstructure and water barrier properties, J. Membr. Sci. 480, 93 (2015).

[25] F. Kremer and A. Schönhals, Broadband Dielectric Spectroscopy (Springer Science \& Business Media, New York, 2012).

[26] C. Dalle-Ferrier, C. Thibierge, C. Alba-Simionesco, L. Berthier, G. Biroli, J.-P. Bouchaud, F. Ladieu, D. L'Hôte, and G. Tarjus, Spatial correlations in the dynamics of glassforming liquids: Experimental determination of their temperature dependence, Phys. Rev. E 76, 041510 (2007).

[27] M. L. Williams, R. F. Landel, and J. D. Ferry, The temperature dependence of relaxation mechanisms in amorphous polymers and other glass-forming liquids, J. Am. Chem. Soc. 77, 3701 (1955).

[28] K. L. Ngai and C. M. Roland, Chemical structure and intermolecular cooperativity: dielectric relaxation results, Macromolecules 26, 6824 (1993).

[29] M. Nakanishi and R. Nozaki, Model of the cooperative rearranging region for polyhydric alcohols, Phys. Rev. E 84, 011503 (2011). 